\title{
Arterial Stiffness Is Significantly Associated With Left Ventricular Diastolic Dysfunction in Patients With Cardiovascular Disease
}

\author{
Takayuki Namba, ${ }^{1}$ MD, Nobuyuki Masaki, ${ }^{1}$ MD, Yuki Matsuo, ${ }^{1}$ MD, Atsushi SAto, ${ }^{1}$ MD, \\ Toyokazu Kimura, ${ }^{1}$ MD, Shunpei HoriI,${ }^{1}$ MD, Risako Yasuda,${ }^{1}$ MD, Hirotaka YadA,,${ }^{1}$ MD, \\ Akio Kawamura, ${ }^{1} \mathrm{MD}$, Bonpei TaKase, ${ }^{2} \mathrm{MD}$, and Takeshi AdachI, ${ }^{1} \mathrm{MD}$
}

\begin{abstract}
SUMMARY
Left ventricular (LV) diastolic dysfunction is considered the main cause of heart failure with preserved ejection fraction $(\mathrm{HFpEF})$. There have been few reports on the correlation between LV diastolic dysfunction and arterial stiffness in patients with clinical cardiovascular disease.

This cross-sectional study enrolled 100 patients (67 men, 33 women; mean age, 70 years). All participants were diagnosed with cardiovascular disease. A total of $89(89 \%)$ patients had coronary artery disease or HF. Patients with reduced EF and valvular disease were excluded. Arterial stiffness was assessed by the cardio-ankle vascular index (CAVI), and LV diastolic dysfunction was estimated using echocardiography. The patients were divided into two groups based on the median value of CAVI. In all patients the ratio of early diastolic transmitral flow velocity to early diastolic mitral annular velocity (E/e') was significantly higher in the high CAVI group than in the low CAVI group $(15.5 \pm 6.4$ versus 12.5 $\pm 2.9, P=0.003)$. In the HF subgroup, E/e' was also significantly higher in the high CAVI group than in the low CAVI group $(17.2 \pm 5.9$ versus $13.0 \pm 3.1, P=0.026)$. In univariate regression analysis, CAVI was significantly associated with E/e' in all patients $(\beta=0.28, P=0.004)$ and in HF patients $(\beta=0.4, P=0.028)$. Also in multivariate analysis, CAVI remained as an independent predictive factor of $\mathrm{E} / \mathrm{e}^{\prime}(\beta=0.252, P=0.037)$.

A high CAVI was independently associated with LV diastolic dysfunction in patients with clinical cardiovascular disease. These results suggested that arterial stiffness contributed to the development of LV diastolic dysfunction. (Int Heart J 2016; 57: 729-735)
\end{abstract}

Key words: Cardio-ankle vascular index, Heart failure with preserved ejection fraction

$I^{\prime}$ Japan and other countries, approximately $40-50 \%$ of patients with heart failure (HF) have preserved left ventricular ejection fraction (LVEF). This is called HF with preserved ejection fraction (HFpEF) and is thought to be mainly caused by LV diastolic dysfunction. ${ }^{1-4)}$ Various factors, including aging, hypertension, diabetes mellitus, chronic kidney disease, metabolic syndrome, and arterial stiffness are considered causes of LV diastolic dysfunction and can be important in the development of HFpEF. ${ }^{5,6)}$ The development of arterial stiffness in HF patients was reported to correlate with poor prognosis; therefore, assessment and management of arterial stiffness are important. ${ }^{7}$ Pulse wave velocity (PWV) has been widely used as a non-invasive index of arterial stiffness. ${ }^{8)}$ Especially in Japan, brachial-ankle PWV (baPWV) is widely measured as a simple index of arterial stiffness. ${ }^{9,10)}$

Cardio-ankle vascular index (CAVI), which was developed in Japan and has less evidence than baPWV, also provides a reproducible index of arterial stiffness, independent of blood pressure (BP). ${ }^{11,12)}$ A correlation between LV diastolic dysfunction and arterial stiffness has been reported, mainly in patients with subclinical cardiovascular disease such as metabolic syndrome. ${ }^{13-19)}$ However, there have been few reports on this correlation in patients with clinical cardiovascular disease and LV diastolic dysfunction.

We examined the correlation between CAVI and multiple parameters, including echocardiographic and laboratory measurements of LV diastolic function and heart failure. We divided patients with clinical cardiovascular diseases, such as coronary artery disease (CAD), HF, and arrhythmia, into two groups based on the median value of CAVI.

\section{METHODS}

Study design and population: In this cross-sectional study, we enrolled 100 patients (67 men, 33 women; mean age, 70 years) diagnosed with clinical cardiovascular disease at the National Defense Medical College Hospital (Tokorozawa, Japan) from

From the Departments of ${ }^{1}$ Cardiology and ${ }^{2}$ Intensive Care Medicine, National Defense Medical College, Tokorozawa, Japan.

Address for correspondence: Takeshi Adachi, MD, Department of Cardiology, National Defense Medical College, 3-2 Namiki, Tokorozawa, Saitama 359-8513, Japan. E-mail: tadachi@ndmc.ac.jp

Received for publication March 4, 2016. Revised and accepted May 5, 2016.

Released in advance online on J-STAGE November 9, 2016.

All rights reserved by the International Heart Journal Association. 
2013 to 2015 . The study protocol complied with the Declaration of Helsinki and the institutional ethics policies.

Clinical cardiovascular diseases included CAD, HF, and arrhythmia. CAD or HF was present in $89 \%$ (89/100) of patients. Continuous medical treatment for clinical cardiovascular disease was required for inclusion in the analysis. Exclusion criteria were as follows: 1) $\mathrm{LVEF}<50 \%$; 2) more than mild valvular disease including aortic valve disease; 3) persistent atrial fibrillation (AF) during measurements; 4) peripheral artery disease (PAD) of the lower limbs, defined by an anklebrachial index $<0.9$; and 5) estimated glomerular filtration rate $(\mathrm{eGFR})<30 \mathrm{~mL} /$ minute.

CAD was defined as the presence of $>75 \%$ stenosis in at least one coronary vessel on an angiogram or a past history of myocardial infarction (MI) or any coronary artery revascularization. HF was defined as the presence of multiple signs and symptoms that were consistent with cardiac decompensation or a past history of hospitalization for HF. All patients with $\mathrm{HF}$ had preserved EF and no valvular disease according to exclusion criteria. Arrhythmia was defined as documentation of paroxysmal AF or ventricular tachycardia. Hypertension was diagnosed by BP above 140/90 $\mathrm{mmHg}$ or the use of BP-lowering medications. Hypercholesterolemia was diagnosed by lowdensity cholesterol (LDL-C) $>140 \mathrm{mg} / \mathrm{dL}$ or the use of LDLlowering medications. Diabetes mellitus (DM) was diagnosed by fasting blood glucose $>126 \mathrm{mg} / \mathrm{dL}$ or the use of anti-diabetes medications.

Plasma LDL-C, triglycerides, high-density cholesterol (HDL-C), glycated hemoglobin, creatinine, and B-type natriuretic peptide (BNP) were measured by standard laboratory tests. The Modification of Diet in Renal Disease equation adjusted for a Japanese population was used to calculate eGFR. ${ }^{20)}$ Cardio-ankle vascular index: CAVI was obtained automati- cally using a vascular screening system (Vasera VS-1000, Fukuda Denshi; Tokyo) based on BP and heart-ankle PWV (haPWV), monitoring of heart sounds, and electrocardiogram. ha PWV was calculated by dividing the distance from the aortic valve to the ankle artery by the sum of the time intervals between aortic valve closure sound and notch of the brachial pulse wave and between the rise of the brachial pulse wave and the ankle pulse wave. CAVI was determined using the following formula,

$$
\mathrm{CAVI}=\mathrm{a}\left[2 \rho /(\mathrm{Ps}-\mathrm{Pd})^{*} \ln (\mathrm{Ps} / \mathrm{Pd})^{*} h a \mathrm{PWV}^{2}\right]+\mathrm{b},
$$

where Ps and Pd are systolic and diastolic BP, respectively; $\rho$ is blood density; and a and b are constants. CAVI was taken as the average of the right and left CAVI values. BP and heart rate (HR) were measured at the time of CAVI measurement.

Echocardiographic assessment: Transthoracic echocardiography was performed at nearly the same time as the CAVI measurement by trained sonographers following a standard protocol. LV dimension, wall thickness, and left atrial diameter (LAD) were measured and LV diastolic dysfunction was assessed by recording LV diastolic inflow on pulsed Doppler echocardiography. Peak early diastolic transmitral flow velocity (E) and peak late diastolic transmitral flow velocity (A) phases of mitral inflow were sampled at the mitral valve leaflet tips from an apical 4-chamber view; from these, the ratio of $\mathrm{E}$ to A (E/A) was calculated. LV myocardial velocity by tissueDoppler imaging was evaluated at the septal mitral valve annulus. The peak early diastolic annular velocity (e') was measured and the ratio of E to e' (E/e') was calculated as an index of LV diastolic dysfunction.

Statistical analysis: Continuous data are expressed as the mean \pm standard deviation (SD) with the $95 \%$ confidential interval for a normal distribution. Comparison of variables in the two groups was performed by Student's $t$-test. Cross-table

Table I. Baseline Characteristics of Patients With Cardiovascular Disease

\begin{tabular}{lcccc}
\hline & Total $(n=100)$ & High CAVI $(n=50)$ & Low CAVI $(n=50)$ & $P$ \\
\hline Age (years) & $70 \pm 8$ & $74 \pm 6$ & $67 \pm 9$ & $<0.001^{*}$ \\
Sex (male/female) & $67(67 \%) / 33(33 \%)$ & $32(64 \%) / 18(36 \%)$ & $35(70 \%) / 15(30 \%)$ & 0.528 \\
Height $(\mathrm{cm})$ & $161 \pm 9.2$ & $159 \pm 8.5$ & $162 \pm 9.7$ & 0.196 \\
Body weight $(\mathrm{kg})$ & $62 \pm 12$ & $59 \pm 12$ & $64 \pm 12$ & 0.074 \\
BMI $\left(\mathrm{kg} / \mathrm{m}^{2}\right)$ & $23.8 \pm 3.6$ & $23.3 \pm 3.4$ & $24.2 \pm 3.7$ & 0.179 \\
Hypertension $(\%)$ & 80 & 80 & 78 & 0.835 \\
Hyperlipidemia $(\%)$ & 73 & 78 & 68 & 0.265 \\
Diabetes mellitus $(\%)$ & 21 & 28 & 14 & 0.087 \\
Number of RF & 1.7 & 1.6 & 1.8 & 0.127 \\
CAD $(\%)$ & 61 & 66 & 56 & 0.310 \\
1VD $(\%)$ & 35 & 32 & 38 & 0.534 \\
2VD $(\%)$ & 11 & 16 & 6 & 0.112 \\
3VD $(\%)$ & 15 & 18 & 12 & 0.406 \\
Prior MI $(\%)$ & 19 & 20 & 18 & 0.801 \\
HF $(\%)$ & 30 & 30 & 30 & 1.000 \\
Arrhythmia $(\%)$ & 16 & 14 & 18 & 0.590 \\
Medication $(\%)$ & & & 48 & \\
Beta blockers & 51 & 53 & 61 & 0.615 \\
ACE-I/ARB & 59 & 57 & 59 & 0.716 \\
CCB & 61 & 84 & 67 & 0.652 \\
Statins & 76 & 27 & 20 & 0.065 \\
Antidiabetic agents & 23 & & & 0.427 \\
\hline
\end{tabular}

"Significant $P$ values are reported. CAVI indicates cardio-ankle vascular index; BMI, body mass index; RF, cardiovascular risk factors; $\mathrm{CAD}$, coronary artery disease; $1 \mathrm{VD}, 2 \mathrm{VD}$, and 3VD, 1-, 2-, and 3-vessel disease, respectively; MI, myocardial infarction; HF, heart failure; ACE-I/ARB, angiotensin-converting enzyme inhibitors and/or angiotensin 2 receptor blockers; and $\mathrm{CCB}$, calcium channel blockers. 
analyses were performed using the chi-square test. Univariate and multivariate regression analyses were performed to identify independent variables from baseline characteristics and laboratory measurements to explain E/e'. In the multivariate analysis, all the independent factors correlating with $\mathrm{E} / \mathrm{e}$ ' $(P<0.1)$ in the univariate analysis were included in a crude model. In all analyses, $P<0.05$ was considered statistically significant. All statistical analyses were performed using JMP version 10.0 (SAS Institute Inc.; Cary, NC, USA).

\section{RESULTS}

Baseline characteristics of the study population: Table I shows the baseline characteristics of the study population. No significant differences were found between the two groups in percentage of males; body mass index; prevalence of hypertension, hyperlipidemia, diabetes mellitus, and number of these cardiovascular risk factors; prevalence of CAD, number of diseased coronary vessels, prior MI, HF and arrhythmia; or medication including angiotensin-converting enzyme inhibitors and/ or angiotensin 2 receptor blockers (ACE-I/ARB) and calcium channel blockers (CCB). However, age was significantly high-

Table II. Laboratory Measurements in Patients With Cardiovascular Disease

\begin{tabular}{lcccc}
\hline & Total $(n=100)$ & High CAVI $(n=50)$ & Low CAVI $(n=50)$ & $P$ \\
\hline CAVI & $8.9 \pm 1.1$ & $9.7 \pm 0.6$ & $8.0 \pm 0.8$ & $<0.001^{*}$ \\
haPWV $(\mathrm{cm} / \mathrm{s})$ & $1602 \pm 322$ & $1700 \pm 197$ & $1465 \pm 242$ & $<0.001^{*}$ \\
sBP $(\mathrm{mmHg})$ & $137 \pm 20$ & $139 \pm 21$ & $136 \pm 17$ & 0.465 \\
dBP $(\mathrm{mmHg})$ & $81 \pm 11$ & $79 \pm 10$ & $83 \pm 11$ & 0.120 \\
$\mathrm{mBP}(\mathrm{mmHg})$ & $100 \pm 12$ & $99 \pm 13$ & $100 \pm 12$ & 0.589 \\
PP $(\mathrm{mmHg})$ & $56 \pm 16$ & $59 \pm 17$ & $53 \pm 14$ & $0.043^{*}$ \\
HR $(\mathrm{bpm})$ & $64 \pm 12$ & $62 \pm 12$ & $67 \pm 12$ & 0.053 \\
LVDd $(\mathrm{cm})$ & $4.7 \pm 0.6$ & $4.7 \pm 0.5$ & $4.7 \pm 0.6$ & 0.812 \\
IVS $(\mathrm{cm})$ & $1.0 \pm 0.2$ & $1.0 \pm 0.2$ & $1.0 \pm 0.2$ & 0.331 \\
PW $(\mathrm{cm})$ & $0.9 \pm 0.1$ & $0.9 \pm 0.1$ & $0.9 \pm 0.2$ & 0.618 \\
LVEF $(\%)$ & $69 \pm 8$ & $70 \pm 8$ & $68 \pm 9$ & 0.116 \\
LAD $(\mathrm{cm})$ & $3.8 \pm 0.5$ & $3.8 \pm 0.5$ & $3.8 \pm 0.5$ & 0.747 \\
E/A & $0.8 \pm 0.3$ & $0.8 \pm 0.3$ & $0.8 \pm 0.3$ & 0.946 \\
e' $(\mathrm{cm} / \mathrm{s})$ & $5.5 \pm 1.7$ & $5.3 \pm 1.7$ & $5.8 \pm 1.7$ & 0.127 \\
E/e' & $14 \pm 5.2$ & $15.5 \pm 6.4$ & $12.5 \pm 2.9$ & $0.003^{*}$ \\
LDL-C (mg/dL) & $93 \pm 25$ & $90 \pm 24$ & $96 \pm 25$ & 0.215 \\
TG $(\mathrm{mg} / \mathrm{dL})$ & $137 \pm 76$ & $135 \pm 65$ & $138 \pm 85$ & 0.853 \\
HDL-C $(\mathrm{mg} / \mathrm{dL})$ & $53 \pm 12$ & $55 \pm 16$ & $58 \pm 16$ & 0.355 \\
HbAlc $(\%)$ & $6.3 \pm 0.8$ & $6.3 \pm 0.9$ & $6.0 \pm 0.7$ & 0.073 \\
eGFR $(\mathrm{mL} / \mathrm{minute})$ & $63 \pm 17$ & $60 \pm 15$ & $67 \pm 18$ & $0.040^{*}$ \\
BNP $(\mathrm{pg} / \mathrm{mL})$ & $70 \pm 112$ & $102 \pm 150$ & $44 \pm 51$ & $0.013^{*}$ \\
\hline
\end{tabular}

"Significant $P$ values are reported. CAVI indicates cardio-ankle vascular index; haPWV, heart-ankle pulse wave velocity; sBP, systolic blood pressure; $\mathrm{dBP}$, diastolic blood pressure; mBP, mean blood pressure; PP, pulse pressure; HR, heart rate; LVDd, left ventricular diastolic diameter; IVS, interventricular septum wall thickness; PW, posterior wall thickness; LVEF, left ventricular ejection fraction; $\mathrm{LAD}$, left atrial diameter; E, peak early diastolic transmitral flow velocity; A, peak late diastolic transmitral flow velocity; e`, peak early diastolic annular velocity; E/A, ratio of E to A; E/e`, ratio of E to e`; LDL-C, low-density lipoprotein cholesterol; TG, triglycerides; eGFR, estimated glomerular filtration rate; and BNP, B-type natriuretic peptide.

Table III. Baseline Characteristics of Patients With HF

\begin{tabular}{lcccc}
\hline & Total $(n=30)$ & High CAVI $(n=15)$ & Low CAVI $(n=15)$ & $P$ \\
\hline Age (years) & $71 \pm 7$ & $72 \pm 5$ & $69 \pm 9$ & 0.256 \\
Sex (male/female) & $14(47 \%) / 16(53 \%)$ & $7(47 \%) / 8(53 \%)$ & $7(47 \%) / 8(53 \%)$ & 1.000 \\
Height $(\mathrm{cm})$ & $158 \pm 10$ & $158 \pm 8$ & $157 \pm 11$ & 0.801 \\
Body weight $(\mathrm{kg})$ & $58 \pm 12$ & $57 \pm 11$ & $59 \pm 13$ & 0.702 \\
BMI $\left(\mathrm{kg} / \mathrm{m}^{2}\right)$ & $23.2 \pm 3.7$ & $22.6 \pm 2.8$ & $23.6 \pm 4.3$ & 0.438 \\
Hypertension $(\%)$ & 83 & 87 & 80 & 0.764 \\
Hyperlipidemia $(\%)$ & 63 & 67 & 60 & 0.716 \\
Diabetes mellitus $(\%)$ & 23 & 40 & 7 & 0.072 \\
Medication $(\%)$ & & & 71 & $0.041^{*}$ \\
$\quad$ Beta blockers & 52 & 33 & 79 & 0.297 \\
ACE-I/ARB & 69 & 60 & 43 & 0.374 \\
CCB & 52 & 60 & 57 & 0.197 \\
Statins & 69 & 80 & 7 & $0.031^{*}$ \\
Antidiabetic agents & 24 & 40 & & 7 \\
\hline
\end{tabular}

"Significant $P$ values are reported. HF indicates heart failure; CAVI, cardio-ankle vascular index; BMI, body mass index; ACE-I/ARB, angiotensin-converting enzyme inhibitors and/or angiotensin 2 receptor blockers; and CCB, calcium channel blockers. 
Table IV. Laboratory Measurements in Patients With HF

\begin{tabular}{|c|c|c|c|c|}
\hline & Total $(n=30)$ & High CAVI $(n=15)$ & Low CAVI $(n=15)$ & $P$ \\
\hline CAVI & $8.9 \pm 1.2$ & $9.9 \pm 0.7$ & $7.9 \pm 0.9$ & $<0.001^{*}$ \\
\hline haPWV $(\mathrm{cm} / \mathrm{s})$ & $1622 \pm 268$ & $1787 \pm 253$ & $1501 \pm 279$ & $0.012^{*}$ \\
\hline $\mathrm{sBP}(\mathrm{mmHg})$ & $141 \pm 22$ & $142 \pm 25$ & $140 \pm 17$ & 0.830 \\
\hline $\mathrm{dBP}(\mathrm{mmHg})$ & $78 \pm 10$ & $79 \pm 10$ & $78 \pm 9$ & 0.929 \\
\hline mBP (mmHg) & $99 \pm 13$ & $100 \pm 14$ & $99 \pm 11$ & 0.865 \\
\hline $\mathrm{PP}(\mathrm{mmHg})$ & $62 \pm 17$ & $63 \pm 19$ & $62 \pm 14$ & 0.824 \\
\hline HR (bpm) & $61 \pm 8$ & $61 \pm 8$ & $62 \pm 9$ & 0.425 \\
\hline LVDd (cm) & $4.9 \pm 0.7$ & $4.7 \pm 0.5$ & $5.1 \pm 0.8$ & 0.104 \\
\hline IVS (cm) & $1.0 \pm 0.3$ & $1.0 \pm 0.2$ & $1.1 \pm 0.3$ & 0.372 \\
\hline $\mathrm{PW}(\mathrm{cm})$ & $1.0 \pm 0.1$ & $1.0 \pm 0.1$ & $1.0 \pm 0.2$ & 1.000 \\
\hline $\operatorname{LVEF}(\%)$ & $66 \pm 9$ & $70 \pm 7$ & $62 \pm 10$ & $0.014^{*}$ \\
\hline $\mathrm{LAD}(\mathrm{cm})$ & $4.0 \pm 0.6$ & $4.0 \pm 0.7$ & $4.0 \pm 0.5$ & 0.921 \\
\hline $\mathrm{E} / \mathrm{A}$ & $0.9 \pm 0.3$ & $0.9 \pm 0.3$ & $0.8 \pm 0.3$ & 0.360 \\
\hline $\mathrm{e}^{\prime}(\mathrm{cm} / \mathrm{s})$ & $5.2 \pm 1.6$ & $5.0 \pm 1.4$ & $5.4 \pm 1.8$ & 0.501 \\
\hline E/e' & $15.1 \pm 5.1$ & $16.7 \pm 6.0$ & $13.0 \pm 3.1$ & $0.026^{*}$ \\
\hline LDL-C (mg/dL) & $104 \pm 25$ & $99 \pm 25$ & $109 \pm 22$ & 0.317 \\
\hline TG (mg/dL) & $126 \pm 50$ & $132 \pm 43$ & $117 \pm 54$ & 0.327 \\
\hline HDL-C (mg/dL) & $60 \pm 17$ & $53 \pm 12$ & $67 \pm 17$ & $0.017^{*}$ \\
\hline HbAlc (\%) & $6.1 \pm 0.7$ & $6.4 \pm 0.8$ & $5.8 \pm 0.6$ & 0.114 \\
\hline eGFR (mL/minute) & $61 \pm 20$ & $58 \pm 18$ & $62 \pm 20$ & 0.769 \\
\hline $\mathrm{BNP}(\mathrm{pg} / \mathrm{mL})$ & $138 \pm 172$ & $205 \pm 217$ & $71 \pm 57$ & $0.027^{*}$ \\
\hline
\end{tabular}

*Significant $P$ values are reported. HF indicates heart failure; CAVI, cardio-ankle vascular index; haPWV, heart-ankle pulse wave velocity; sBP, systolic blood pressure; dBP, diastolic blood pressure; mBP, mean blood pressure; PP, pulse pressure; $\mathrm{HR}$, heart rate; LVDd, left ventricular diastolic diameter; IVS, interventricular septum wall thickness; PW, posterior wall thickness; LVEF, left ventricular ejection fraction; LAD, left atrial diameter; E, peak early diastolic transmitral flow velocity; A, peak late diastolic transmitral flow velocity; e', peak early diastolic annular velocity; E/A, ratio of E to A; E/e', ratio of E to e ; LDL-C, low-density lipoprotein cholesterol; TG, triglycerides; eGFR, estimated glomerular filtration rate; and BNP, B-type natriuretic peptide.

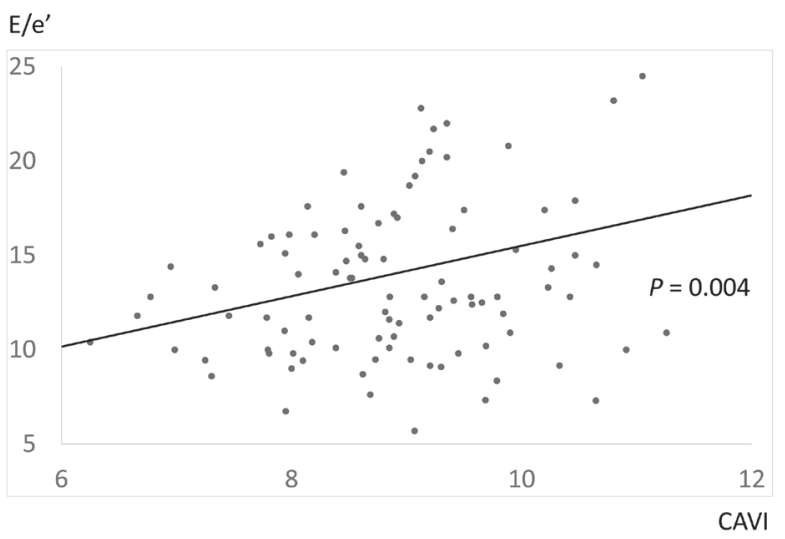

Figure 1. Correlation between cardio-ankle vascular index (CAVI) and the ratio of early diastolic transmitral flow velocity to early diastolic mitral annular velocity (E/e') in all patients.

er in the high CAVI group than in the low CAVI group. Laboratory measurements and echocardiographic characteristics: Table II shows the laboratory measurements and echocardiographic characteristics of the patients according to CAVI value. E/e', pulse pressure, and BNP were significantly higher in the high CAVI group than in the low CAVI group. The high CAVI group had a lower eGFR. Systolic and diastolic BP, HR, LV diastolic diameter, LV wall thickness, E/A, and LVEF were similar between the two groups.

Subgroup analysis of HF patients: There were 30 patients with HF. The percentage of HF patients who were hypertensive was $83 \%$. As in the overall analysis, no significant differences were found between the high and low CAVI groups in

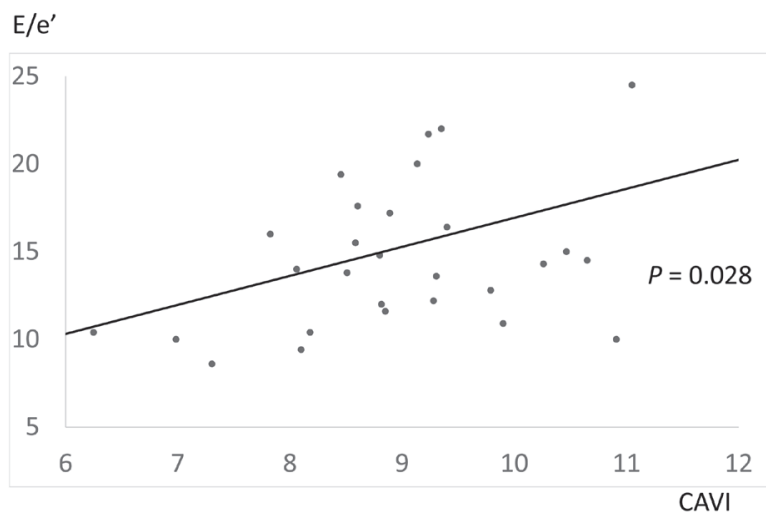

Figure 2. Correlation between CAVI and E/e' in HF patients.

percentage of males; body mass index; or prevalence of hypertension, hyperlipidemia, and diabetes mellitus. In this subgroup analysis, age was not significantly different between the two groups (Table III). Table IV shows the laboratory measurements and echocardiographic characteristics of HF patients according to the CAVI value. Compared with the low CAVI group, the high CAVI group had significantly higher E/e', LVEF, and BNP. HDL-C was lower in the high CAVI group. Systolic and diastolic BP, HR, LV diastolic diameter, LV wall thickness, and LAD were not different between the two groups.

Regression analysis: In univariate analysis, the CAVI was significantly associated with E/e' in all patients $(\beta=0.28, P=$ 0.004) (Figure1) and in HF patients $(\beta=0.4, P=0.03)$ (Figure 2 ). Table $V$ shows the results of regression analysis for predic- 
Table V. Regression Analysis for Prediction of E/e' in Patients With Cardiovascular Disease

\begin{tabular}{|c|c|c|c|c|}
\hline \multirow{2}{*}{ Total $(n=100)$} & \multicolumn{2}{|c|}{ Univariate } & \multicolumn{2}{|c|}{ Multivariate } \\
\hline & $\beta$ & $P$ & $\beta$ & $P$ \\
\hline Age (years) & 0.268 & $0.007^{*}$ & 0.087 & 0.499 \\
\hline Sex $($ male $=1$, female $=0)$ & -0.148 & 0.143 & & \\
\hline BMI $\left(\mathrm{kg} / \mathrm{m}^{2}\right)$ & -0.03 & 0.773 & & \\
\hline Hypertension (yes $=1$, no $=0$ ) & 0.203 & $0.043^{*}$ & 0.143 & 0.196 \\
\hline Hyperlipidemia $($ yes $=1$, no $=0$ ) & -0.004 & 0.972 & & \\
\hline Diabetes mellitus $($ yes $=1$, no $=0$ ) & 0.126 & 0.212 & & \\
\hline CAD $($ yes $=1$, no $=0)$ & -0.004 & 0.968 & & \\
\hline Prior MI (yes = 1, no $=0)$ & 0.006 & 0.952 & & \\
\hline $\mathrm{HF}($ yes $=1$, no $=0)$ & 0.139 & 0.168 & & \\
\hline Arrhythmia (yes = 1, no = 0) & -0.004 & 0.965 & & \\
\hline \multicolumn{5}{|l|}{ Medication } \\
\hline Beta blockers $($ yes $=1$, no $=0)$ & 0.037 & 0.723 & & \\
\hline ACE-I/ARB $($ yes $=1$, no $=0)$ & 0.037 & 0.723 & & \\
\hline CCB $($ yes $=1$, no $=0)$ & 0.127 & 0.219 & & \\
\hline Statins $($ yes $=1$, no $=0)$ & -0.008 & 0.938 & & \\
\hline Antidiabetic agents (yes $=1$, no $=0$ ) & 0.077 & 0.458 & & \\
\hline CAVI & 0.284 & $0.004^{*}$ & 0.252 & $0.037^{*}$ \\
\hline sBP $(\mathrm{mmHg})$ & 0.19 & 0.058 & 0.241 & 0.211 \\
\hline $\mathrm{dBP}(\mathrm{mmHg})$ & 0.07 & 0.487 & & \\
\hline $\mathrm{PP}(\mathrm{mmHg})$ & 0.186 & 0.064 & -0.157 & 0.448 \\
\hline HR (bpm) & -0.083 & 0.411 & & \\
\hline $\operatorname{LVEF}(\%)$ & 0.14 & 0.163 & & \\
\hline LDL-C (mg/dL) & 0.111 & 0.278 & & \\
\hline $\mathrm{TG}(\mathrm{mg} / \mathrm{dL})$ & -0.068 & 0.513 & & \\
\hline HDL-C (mg/dL) & 0.176 & 0.088 & 0.211 & 0.050 \\
\hline $\mathrm{HbA1c}(\%)$ & 0.076 & 0.491 & & \\
\hline eGFR (mL/minute) & -0.116 & 0.254 & & \\
\hline $\mathrm{BNP}(\mathrm{pg} / \mathrm{mL})$ & 0.198 & 0.062 & 0.110 & 0.345 \\
\hline
\end{tabular}

${ }^{*}$ Significant $P$ values are reported. BMI indicates body mass index; CAD, coronary artery disease; MI, myocardial infarction; HF, heart failure; ACE-I/ARB, angiotensin-converting enzyme inhibitors and/or angiotensin 2 receptor blockers; CCB, calcium channel blockers; CAVI, cardio-ankle vascular index; sBP, systolic blood pressure; dBP, diastolic blood pressure; PP, pulse pressure; HR, heart rate; LVEF, left ventricular ejection fraction; LDL-C, low-density lipoprotein cholesterol; TG, triglycerides; eGFR, estimated glomerular filtration rate; and BNP, B-type natriuretic peptide.

tion of E/e' in patients with cardiovascular disease. The factors other than CAVI that correlated with E/e' were age, prevalence of HT, systolic BP, pulse pressure, HDL-C, and BNP. In a crude model, CAVI remained as an independent predictive factor of E/e' $(\beta=0.252, P=0.037)$, but age and other factors did not.

\section{Discussion}

The incidence of patients with HFpEF is constantly increasing, but therapeutic strategies are limited. Therefore, identification of the pathophysiologic factors in HFpEF is necessary to formulate new therapeutic strategies. CAVI is a typical parameter of arterial stiffness that is independent of BP. In this study, we investigated the relationship between CAVI and echocardiographic parameters in patients with clinical cardiovascular disease. The results showed that CAVI was positively correlated with E/e' in all patients and in the HF subgroup. These data suggested a strong correlation between arterial stiffness and LV diastolic dysfunction, independent of BP.

The contribution of elevated LV afterload to the development of LV diastolic dysfunction has been considered. ${ }^{21)}$ From what is known as the Windkessel effect, when blood is ejected from the heart to the aorta, the transient expansion of the aorta transmits pulse waves to peripheral arteries. This pulse wave is reflected at the bifurcation of the common iliac artery, whereas the retrograde reflection pulse wave amplifies arterial BP when it returns to the ascending aorta. The return of the reflection pulse wave to the ascending aorta happens during diastole when PWV is slow and during late systole when PWV is fast due to arterial stiffness. This change in loading sequence amplifies systolic BP and increases pulse pressure. ${ }^{22,23)}$ As a result, $\mathrm{LV}$ afterload is increased. In addition, this change reduces the time constant $\tau$, an indicator of LV relaxation, which is a component of LV diastolic function and significantly correlates with the development of LV diastolic dysfunction. ${ }^{24)}$ When PWV is fast, the arrival of the reflection pulse wave during late systole causes a reduction in diastolic pressure and may correlate with LV diastolic dysfunction due to endomyocardial ischemia secondary to a reduction in coronary perfusion pressure. ${ }^{25)}$ In the present study, we employed CAVI calculated by inserting haPWV, because it reflects stiffness of the whole arterial system from the aortic root to the lower extremities and might be more appropriate to estimate the part of LV afterload.

Systemic vascular resistance (SVR) is widely used as an index of LV afterload. Thus, vasodilator treatment is administered to reduce SVR in cases of acute decompensated HF and to prevent elevation of SVR and LV afterload mismatch in chronic HF. However, as a component of LV afterload, arterial stiffness is important along with SVR, especially in elderly patients. ${ }^{5,626-29)}$ Therefore, it may not be appropriate to prevent the 
development of LV diastolic dysfunction by reduction of SVR alone.

Some reports have discussed the correlation between arterial stiffness and LV diastolic dysfunction, mainly in subclinical patients with cardiovascular risk factors, and in patients with clinical cardiovascular diseases including $\mathrm{CAD}$ or $\mathrm{HF}^{13-19)}$ In the present study, all patients had clinical cardiovascular diseases. In particular, approximately $90 \%$ of the patients enrolled had CAD or HF and required continuous medical treatment. Many of the patients already had stiff arteries and LV diastolic dysfunction, so the average values of CAVI (8.9) and E/e' (14.0) were high. Based on our results, patients with possible LV diastolic dysfunction may require therapeutic intervention for arterial stiffness. However, a medical intervention for arterial stiffness has not been established. There have been several reports on possible effective pharmacologic therapies, such as ACE-I/ARB, CCB, statins, and sarpogrelate hydrochloride, and non-pharmacologic therapies, such as exercise and continuous positive airway pressure. ${ }^{30-39)}$ Further studies are needed to establish indicators and therapeutic methods for arterial stiffness.

There are several limitations in our study. First, the sample size was small. Second, patients with reduced EF (LVEF < $50 \%$ ), more than mild valvular disease, ankle-brachial index $<$ 0.9 , persistent $\mathrm{AF}$, and $\mathrm{eGFR}<30 \mathrm{~mL} /$ minute were excluded, so the applicability of our results to such patients is unknown. Third, the study did not focus on patients with HF because we aimed to ascertain the general relationship between CAVI and echocardiographic data as an initial study.

In conclusion, a high CAVI value was independently associated with LV diastolic dysfunction in patients with clinical cardiovascular disease. The results suggest that arterial stiffness contributed to the development of LV diastolic dysfunction. The measurement of CAVI might be an important application for the risk of HFpEF.

\section{Disclosure}

None.

\section{REFERENCES}

1. Tsuchihashi-Makaya M, Hamaguchi S, Kinugawa S, et al. Characteristics and outcomes of hospitalized patients with heart failure and reduced vs preserved ejection fraction. Report from the Japanese Cardiac Registry of Heart Failure in Cardiology (JCARECARD). Circ J 2009; 73: 1893-900.

2. Go AS, Mozaffarian D, Roger VL, et al. Heart disease and stroke statistics--2013 update: a report from the American Heart Association. Circulation 2013; 127: e6-245.

3. Zile MR, Baicu CF, Gaasch WH. Diastolic heart failure--abnormalities in active relaxation and passive stiffness of the left ventricle. N Engl J Med 2004; 350: 1953-9.

4. Owan TE, Hodge DO, Herges RM, Jacobsen SJ, Roger VL, Redfield MM. Trends in prevalence and outcome of heart failure with preserved ejection fraction. N Engl J Med 2006; 355: 251-9.

5. Kawaguchi M, Hay I, Fetics B, Kass DA. Combined ventricular systolic and arterial stiffening in patients with heart failure and preserved ejection fraction: implications for systolic and diastolic reserve limitations. Circulation 2003; 107: 714-20.

6. Lam CS, Roger VL, Rodeheffer RJ, et al. Cardiac structure and ventricular-vascular function in persons with heart failure and preserved ejection fraction from Olmsted County, Minnesota. Circulation 2007; 115: 1982-90.

7. Meguro T, Nagatomo Y, Nagae A, et al. Elevated arterial stiffness evaluated by brachial-ankle pulse wave velocity is deleterious for the prognosis of patients with heart failure. Circ J 2009; 73: 67380.

8. Asmar R, Benetos A, Topouchian J, et al. Assessment of arterial distensibility by automatic pulse wave velocity measurement. Validation and clinical application studies. Hypertension 1995; 26 : 485-90.

9. Yamashina A, Tomiyama H, Takeda K, et al. Validity, reproducibility, and clinical significance of noninvasive brachial-ankle pulse wave velocity measurement. Hypertens Res 2002; 25: 359-64

10. Matsui Y, Kario K, Ishikawa J, Eguchi K, Hoshide S, Shimada K. Reproducibility of arterial stiffness indices (pulse wave velocity and augmentation index) simultaneously assessed by automated pulse wave analysis and their associated risk factors in essential hypertensive patients. Hypertens Res 2004; 27: 851-7.

11. Yambe T, Yoshizawa M, Saijo Y, et al. Brachio-ankle pulse wave velocity and cardio-ankle vascular index (CAVI). Biomed Pharmacother 2004; 58: S95-8. (Review)

12. Shirai K, Utino J, Otsuka K, Takata M. A novel blood pressure-independent arterial wall stiffness parameter; cardio-ankle vascular index (CAVI). J Atheroscler Thromb 2006; 13: 101-7.

13. Satoh N, Shimatsu A, Kato Y, et al. Evaluation of the cardio-ankle vascular index, a new indicator of arterial stiffness independent of blood pressure, in obesity and metabolic syndrome. Hypertens Res 2008; 31: 1921-30.

14. Sakane K, Miyoshi T, Doi M, et al. Association of new arterial stiffness parameter, the cardio-ankle vascular index, with left ventricular diastolic function. J Atheroscler Thromb 2008; 15: 261-8.

15. Masugata H, Senda S, Goda F, et al. Tissue Doppler echocardiography for predicting arterial stiffness assessed by cardio-ankle vascular index. Tohoku J Exp Med 2009; 217: 139-46.

16. Mizuguchi $\mathrm{Y}$, Oishi $\mathrm{Y}$, Tanaka $\mathrm{H}$, et al. Arterial stiffness is associated with left ventricular diastolic function in patients with cardiovascular risk factors: early detection with the use of cardio-ankle vascular index and ultrasonic strain imaging. J Card Fail 2007; 13: 744-51.

17. Abhayaratna WP, Barnes ME, O'Rourke MF, et al. Relation of arterial stiffness to left ventricular diastolic function and cardiovascular risk prediction in patients $>$ or $=65$ years of age. Am J Cardiol 2006; 98: 1387-92.

18. Eren M, Gorgulu S, Uslu N, Celik S, Dagdeviren B, Tezel T. Relation between aortic stiffness and left ventricular diastolic function in patients with hypertension, diabetes, or both. Heart 2004; 90 : $37-43$.

19. Kim G, Kim JH, Moon KW, et al. The Relationships Between the Arterial Stiffness Index Measured at the Radial Artery and Left Ventricular Diastolic Dysfunction in Asymptomatic High Risk Patients Without Atherosclerotic Cardiovascular Disease. Int Heart J 2016; 57: 73-9.

20. Imai E, Horio M, Nitta K, et al. Estimation of glomerular filtration rate by the MDRD study equation modified for Japanese patients with chronic kidney disease. Clin Exp Nephrol 2007; 11: 41-50.

21. Leite-Moreira AF, Correia-Pinto J, Gillebert TC. Afterload induced changes in myocardial relaxation: a mechanism for diastolic dysfunction. Cardiovasc Res 1999; 43: 344-53.

22. Fujimoto N, Onishi K, Dohi K, et al. Hemodynamic characteristics of patients with diastolic heart failure and hypertension. Hypertens Res 2008; 31: 1727-35.

23. O'Rourke MF, Hashimoto J. Mechanical factors in arterial aging: a clinical perspective. J Am Coll Cardiol 2007; 50: 1-13. (Review)

24. Hori M, Inoue $\mathrm{M}$, Kitakaze $\mathrm{M}$, et al. Loading sequence is a major determinant of afterload-dependent relaxation in intact canine heart. Am J Physiol 1985; 249: H747-54.

25. Mottram PM, Haluska BA, Leano R, Carlier S, Case C, Marwick TH. Relation of arterial stiffness to diastolic dysfunction in hypertensive heart disease. Heart 2005; 91: 1551-6. 
26. Kelly RP, Tunin R, Kass DA. Effect of reduced aortic compliance on cardiac efficiency and contractile function of in situ canine left ventricle. Circ Res 1992; 71: 490-502.

27. Melenovsky V, Borlaug BA, Rosen B, et al. Cardiovascular features of heart failure with preserved ejection fraction versus nonfailing hypertensive left ventricular hypertrophy in the urban Baltimore community: the role of atrial remodeling/dysfunction. J Am Coll Cardiol 2007; 49: 198-207.

28. Borlaug BA, Kass DA. Ventricular-vascular interaction in heart failure. Cardiol Clin 2011; 29: 447-59.

29. Goto T, Ohte N, Fukuta H, Wakami K, Tani T, Kimura G. Relationship between effective arterial elastance, total vascular resistance, and augmentation index at the ascending aorta and left ventricular diastolic function in older women. Circ J 2013; 77: 123-9.

30. Cavalcante JL, Lima JA, Redheuil A, Al-Mallah MH. Aortic stiffness: current understanding and future directions. J Am Coll Cardiol 2011; 57: 1511-22. (Review)

31. Mitchell GF, Dunlap ME, Warnica W, et al. Long-term trandolapril treatment is associated with reduced aortic stiffness: the prevention of events with angiotensin-converting enzyme inhibition hemodynamic substudy. Prevention of Events With AngiotensinConverting Enzyme Inhibition Investigators. Hypertension 2007; 49: 1271-7.

32. Bokuda K, Ichihara A, Sakoda M, et al. Blood pressure-independent effect of candesartan on cardio-ankle vascular index in hypertensive patients with metabolic syndrome. Vasc Health Risk Man- ag 2010; 6: 571-8.

33. Ting CT, Chen CH, Chang MS, Yin FC. Short- and long-term effects of antihypertensive drugs on arterial reflections, compliance, and impedance. Hypertension 1995; 26: 524-30.

34. Williams B, Lacy PS, Thom SM, et al. Differential impact of blood pressure-lowering drugs on central aortic pressure and clinical outcomes: principal results of the Conduit Artery Function Evaluation (CAFE) study. Circulation 2006; 113: 1213-25.

35. Ichihara A, Hayashi M, Ryuzaki M, Handa M, Furukawa T, Saruta T. Fluvastatin prevents development of arterial stiffness in haemodialysis patients with type 2 diabetes mellitus. Nephrol Dial Transplant 2002; 17: 1513-7.

36. Nagayama D, Ohira M, Saiki A, Shirai K, Tatsuno I. Sarpogrelate hydrochloride decreases cardio-ankle vascular index accompanied by increased serum lipoprotein lipase mass in type 2 diabetic patients. Int Heart J 2014; 55: 337-41.

37. Kakiyama T, Matsuda M, Koseki S. Effect of physical activity on the distensibility of the aortic wall in healthy males. Angiology 1998; 49: 749-57.

38. Vaitkevicius PV, Fleg JL, Engel JH, et al. Effects of age and aerobic capacity on arterial stiffness in healthy adults. Circulation 1993; 88: 1456-62.

39. Phillips CL, Yee B, Yang Q, et al. Effects of continuous positive airway pressure treatment and withdrawal in patients with obstructive sleep apnea on arterial stiffness and central BP. Chest 2008; 134: 94-100. 